\title{
The strato-rotational instability of Taylor-Couette and Keplerian flows
}

\author{
S. LE DIZÈ S† AND X. RIEDINGER \\ IRPHE, UMR 6594 CNRS, 49 rue F. Joliot Curie, F-13013 Marseille, France \\ (Received 23 September 2009; revised 30 April 2010; accepted 2 May 2010; \\ first published online 23 August 2010)
}

The linear inviscid stability of two families of centrifugally stable rotating flows in a stably stratified fluid of constant Brunt-Väisälä frequency $N$ is analysed by using numerical and asymptotic methods. Both Taylor-Couette and Keplerian angular velocity profiles $\Omega_{T C}=(1-\mu) / r^{2}+\mu$ and $\Omega_{K}=(1-\lambda) / r^{2}+\lambda / r^{3 / 2}$ are considered between $r=1$ (inner boundary) and $r=d>1$ (outer boundary, or without boundary if $d=\infty$ ). The stability properties are obtained for flow parameters $\lambda$ and $\mu$ ranging from 0 to $+\infty$, and different values of $d$ and $N$. The effect of the gap size is analysed first. By considering the potential flow $(\lambda=\mu=0)$, we show how the instability associated with a mechanism of resonance for finite-gap changes into a radiative instability when $d \rightarrow \infty$. Numerical results are compared with large axial wavenumber results and a very good agreement is obtained. For infinite gap $(d=\infty)$, we show that the most unstable modes are obtained for large values of the azimuthal wavenumber for all $\lambda$ and $\mu$. We demonstrate that their properties can be captured by performing a local analysis near the inner cylinder in the limit of both large azimuthal and axial wavenumbers. The effect of the stratification is also analysed. We show that decreasing $N$ is stabilizing. An asymptotic analysis for small $N$ is also performed and shown to capture the properties of the most unstable mode of the potential flow in this limit.

Key words: stratified flows, vortex instability, waves in rotating fluids

\section{Introduction}

The stability of rotating flows has been the subject of an enormous amount of works as it concerns almost all the domains of applications of fluid mechanics. Simple inviscid stability criteria have been obtained for axisymmetric rotating flows described by their azimuthal velocity profile $V(r)$. It is known (Hopfinger \& van Heijst 1993) that a rotating flow is stable with respect to the centrifugal instability if $\mathrm{d}(r V)^{2} / \mathrm{d} r>0$ for all $r$, and stable with respect to the shear instability if its vorticity $r^{-1} \mathrm{~d}(r V) / \mathrm{d} r$ has no local extremum at any radial location $r>0$. These stability criteria are not affected by a (stable) linear stratification along the vortex axis, but a new instability, called the strato-rotational instability (SRI) is however possible. This instability has been predicted and observed in Taylor-Couette systems (Withjack \& Chen 1974; Molemaker, McWilliams \& Yavneh 2001; Shalybkov \& Rüdiger 2005; Le Bars \& Le Gal 2007). Using a small-gap approach, Yavneh, McWilliams \& Molemaker (2001) showed that the instability mechanism can be understood as 
a resonance of boundary trapped waves. Here we analyse the instability by using a different asymptotic approach, based on Wentzel-Kramers-Brillouin-Jeffreys (WKBJ) approximations for large axial wavenumbers (Le Dizès \& Lacaze 2005), which allows us to consider large-gap systems as well as radially open flows. This method has been used by Billant \& Gallaire (2005) for the centrifugal instability in a homogeneous Taylor-Couette flow. Lamb-Oseen and Rankine vortices in a stratified fluid were considered in Le Dizès \& Billant (2009) and Billant \& Le Dizès (2009). They were able to show that the instability is directly associated with the emission of internal gravity waves, in agreement with earlier results by Schecter \& Montgomery (2004). Froude and Reynolds numbers effects were further analysed in Riedinger, Le Dizès \& Meunier (2010a) for the Lamb-Oseen vortex. Experimental results were also obtained in Riedinger, Meunier \& Le Dizès (2010b). In Riedinger et al. (2010a), the authors were able to show that two types of helical modes $(m=1)$ could become the most unstable. The first type corresponds, for small axial wavenumbers, to a displacement mode. For large Reynolds numbers, it is the most unstable mode for small Froude number $(F<2)$. The second type of mode is a so-called ring mode, which is the first of an infinite series of modes. In the geophysical context, this mode corresponds to a vortex Rossby wave with an internal gravity wave structure far from the vortex. It was shown in Le Dizès \& Billant (2009) that these modes are well described by the WKBJ approach.

Our first goal is here to quantify the effect of angular velocity variations on the characteristics of the instability. The flow domain will be limited by a rigid cylindrical boundary at $r=1$. As a consequence, the displacement mode found for the LambOseen vortex in a fully open domain will not be present. However, similar ring modes will be found. The second goal is to study the effect of the outer boundary and to analyse how the transition between the SRI in a Taylor-Couette system and the radiative instability in an open domain occurs. The third goal is to provide some quantitative results on the variation of the stability properties with respect to the azimuthal wavenumber and the strength of the stratification.

The paper is organized as follows. The framework is briefly presented in $\S 2$. We first consider a potential flow and analyse for such a flow successively the effect of the outer boundary $(\S 3)$, the dependence on the stratification $(\S 4)$, and the variation of the stability properties with respect to the azimuthal wavenumber $(\S 5)$. Also in $\S 5$ an asymptotic analysis for large $m$ is performed. In $\S \S 6$ and 7, numerical results are obtained for the Taylor-Couette and Keplerian flows in the larger-gap limit and compared with asymptotic results. In the appendices, two asymptotic analyses are performed for the potential Taylor-Couette flow.

\section{Base flow and perturbation equations}

We shall consider two families of rotating flows defined by their azimuthal velocity profile:

$$
\begin{aligned}
& V_{T C}=\frac{1-\mu}{r}+\mu r, \quad \text { with } 0 \leqslant \mu \quad \text { for } 1 \leqslant r \leqslant d \text {, } \\
& V_{K}=\frac{1-\lambda}{r}+\frac{\lambda}{r^{1 / 2}}, \quad \text { with } 0 \leqslant \lambda \text { for } 1 \leqslant r \leqslant d \text {, }
\end{aligned}
$$

where $r$ is the radial cylindrical coordinate. Each family can be considered as a perturbation of the potential flow obtained for $\mu=\lambda=0$. The first family $\left(V_{T C}\right)$ corresponds to the classical Taylor-Couette flow profile. It is an exact solution of the 
Navier-Stokes equations. The second family is a model which has been introduced to describe thin accretion disks (Dubrulle et al. 2005a). It corresponds to a pure Keplerian flow for $\lambda=1$. This second family of flows is a solution of the NavierStokes equations if we add the radial velocity component $U=-3 v /(2 r)$ where $v$ is the kinematic viscosity. Here, we shall not consider viscous effects and thus neglect such a radial flow.

For both flows, the fluid is assumed to be stably stratified along the $z$-axis of rotation with a constant Brunt-Väisälä frequency $N=\sqrt{-(g / \rho) \partial \rho / \partial z}$. The deformations of the isopycnals and isobars induced by the rotation can be easily calculated from the cyclostrophic and hydrostatic balances for both velocity profiles. However, the effect of these deformations on the perturbations will not be considered as a Boussinesq approximation will be used in the following. Moreover, the diffusion of the density will be also neglected. With the normalization chosen for the base flow, $N$ can be considered as the inverse of an horizontal Froude number. All values of the Froude number will be considered.

Note that for negative parameters $\lambda$ and $\mu$, both flows are unstable with respect to the centrifugal instability. This instability is much stronger than the instability that we shall describe here. For this reason, we have limited the study to positive values of $\mu$ and $\lambda$.

For large gap, the Taylor-Couette flow mimics the flow around a vortex in a rotating environment. The Rossby number for this vortex is related to $\mu$ by the relation $R o=(1-\mu) / \mu$. Thus, the parameter range $0<\mu<1$ corresponds to cyclonic vortices whereas the range $\mu>1$ describes weak anticyclones of Rossby number $|R o|$ smaller than 1.

In the accretion disk literature (see Goldreich \& Lynden-Bell 1965; Narayan, Goldreich \& Goodman 1987), the Keplerian flow $(\lambda=1)$ has often been modelled by a small-gap Taylor-Couette flow with $\mu=1 / 4$. This corresponds to the local approximation of the Keplerian flow for which both flows possess the same shearto-rotation ratio $\Omega^{\prime} / O m$ ( $\Omega$ being the angular velocity) equal to $-3 / 2$. The stability properties of the flow in this small-gap limit have been obtained in Yavneh et al. (2001) and Dubrulle et al. (2005b). We shall see that they sensibly differ from those obtained for an unbounded Keplerian flow.

We consider linear normal mode perturbations for the velocity $\tilde{\boldsymbol{u}}=(\tilde{u}, \tilde{v}, \tilde{w})$, pressure $\tilde{p}$ and density $\tilde{\rho}$ of the form

$$
(\tilde{\boldsymbol{u}}, \tilde{p}, \tilde{\rho})(r, \theta, z, t)=(\boldsymbol{u}, p, \rho)(r) \mathrm{e}^{\mathrm{i} k z+\mathrm{i} m \theta-\mathrm{i} \omega t}
$$

where $k$ and $m$ are axial and azimuthal wavenumbers and $\omega$ is the frequency. If we neglect the diffusion processes and use the Boussinesq approximation, the linearized equations for these perturbations become

$$
\begin{gathered}
-\mathrm{i} \Phi u-2 \Omega v=-\frac{\mathrm{d} p}{\mathrm{~d} r}, \\
-\mathrm{i} \Phi v+\zeta u=-\frac{\mathrm{i} m p}{r}, \\
-\mathrm{i} \Phi w=-\mathrm{i} k p-N^{2} \rho, \\
-\mathrm{i} \Phi \rho=w, \\
\frac{1}{r} \frac{\mathrm{d}(r u)}{\mathrm{d} r}+\frac{\mathrm{i} m v}{r}+\mathrm{i} k w=0,
\end{gathered}
$$



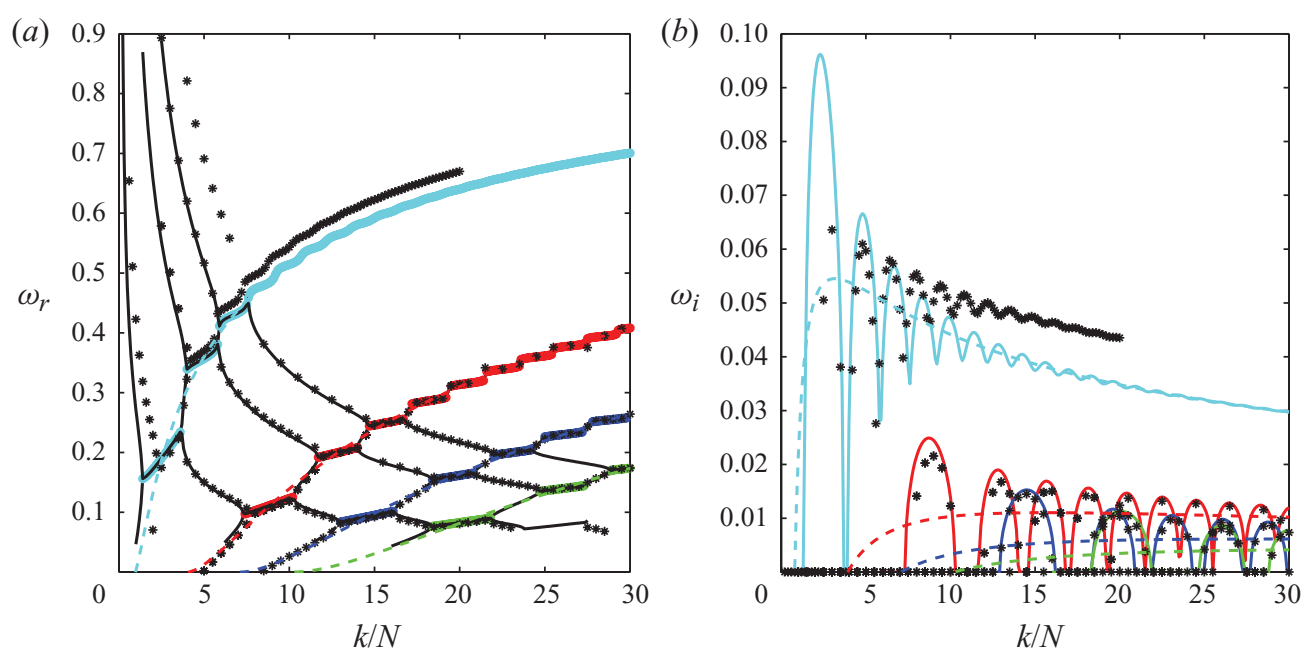

FIGURE 1. Frequency $\omega_{r}(a)$ and growth rate $\omega_{i}(b)$ versus the rescaled axial wavenumber $k / N$ of the helical mode $m=1$ for $\mu=0, N=5$ and $d=5$. Symbols: numerical results. Solid lines: WKBJ results for $d=5$ (defined in (A 8)). The dashed lines are the WKBJ results for $d=\infty$ (see (A 7)). The colours in $(b)$ correspond to the frequencies indicated with the same colour in (a) and for which $\omega_{i}>0$.

where $\Phi(r)=\omega-m \Omega(r)$ is the Lagrangian frequency, and $\Omega(r)=V / r$ and $\zeta(r)=V^{\prime}+$ $V / r$ are the angular velocity and the axial vorticity of the flow. These equations can be reduced (Le Dizès \& Billant 2009) to a single equation for the amplitude $\psi(r)=r u(r)$ :

$$
\frac{\mathrm{d}^{2} \psi}{\mathrm{d} r^{2}}-\left(\frac{1}{r}+\frac{G^{\prime}}{G}\right) \frac{\mathrm{d} \psi}{\mathrm{d} r}+\left[\frac{m \zeta}{r \Phi}\left(\frac{\zeta^{\prime}}{\zeta}-\frac{G^{\prime}}{G}-\frac{2}{r}\right)+\frac{k^{2}\left(\Phi^{2}-2 \zeta \Omega\right)}{N^{2}-\Phi^{2}}-\frac{m^{2}}{r^{2}}\right] \psi=0,
$$

where $G(r)=m^{2} / r^{2}+k^{2} \Phi^{2} /\left(\Phi^{2}-N^{2}\right)$. By applying vanishing boundary conditions on $u$, or a condition of outward radiation if $d=\infty$, we obtain an eigenvalue problem for $\omega$. This problem is solved numerically by using either a shooting method with (2.4) or a pseudospectral collocation method with the system (2.3). Details on the numerical techniques can be found in Riedinger et al. (2010a). For each flow, we have three base flow parameters $\mu$ or $\lambda, N$ and $d$ and two perturbation parameters $m$ and $k$.

\section{Effect of the finite gap size}

In this section, we want to analyse the effect of the parameter $d$, in order to understand the limit $d \rightarrow \infty$. For this purpose, we shall focus on the potential TaylorCouette flow which corresponds to the flow profile obtained for $\mu=0$ or $\lambda=0$.

Small-gap configurations have been considered by Yavneh et al. (2001) who have shown that the flow is unstable when the fluid is sufficiently stratified. In figure 1, results for a large-gap configuration $(d=5)$ are plotted. The two series of frequency curves shown in figure $1(a)$ correspond to the trapped modes already mentioned by Yavneh et al. (2001). The different branches correspond to the number of radial oscillations of the modes in the region where they exist. Resonance occurs at the crossing point of these curves. Around each crossing point, one of the two modes becomes unstable. The growth rate associated with each resonance is plotted in figure $1(b)$. In these figures are also plotted the theoretical predictions obtained by a WKBJ analysis in the limit of large axial wavenumbers. This theory, which is presented 

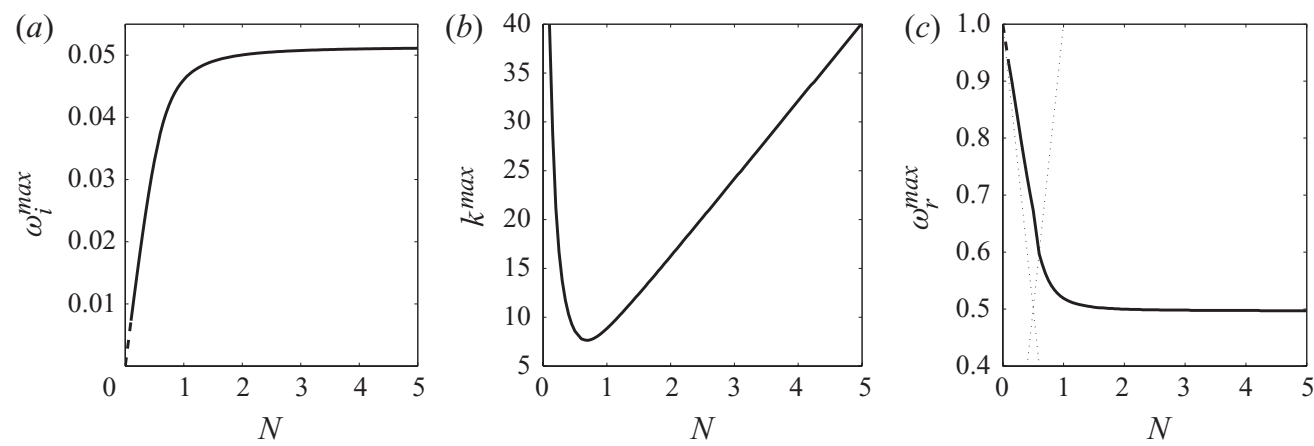

Figure 2. Characteristics of the most unstable helical mode $(m=1)$ for an unbounded potential Taylor-Couette flow $(\mu=0, d=\infty)$. (a) Maximum growth rate; $(b)$ wavenumber; $(c)$ frequency. The dotted lines are the limits of the small $N$ domains for which a critical point exists in the eigenmode structure. These lines are given by $\omega=N$ and $\omega=1-N$ for $r_{c}^{+}$and $r_{c}^{-}$respectively.

in Appendix A, has already been used for non-stratified vortices (Le Dizès \& Lacaze 2005) and stratified columnar vortices (Billant \& Le Dizès 2009; Le Dizès \& Billant 2009). For the potential Taylor-Couette flow $(\mu=0)$, it provides an explicit dispersion relation as a function of the parameters $N, k$ and $m$. In figure 1 , both the theoretical predictions for $d=5$ and $d=\infty$ have been plotted. Note that for the bounded case $(d=5)$, theory and numerics agree both for the frequency and the growth rate. For $d=\infty$, one can notice in figure $1(a)$ that one part of the spectrum has disappeared: the modes localized near the outer cylinder, and which correspond to the branches with a decreasing behaviour with respect to $k / N$, are no longer present. Only the modes close to the inner cylinder remain. The frequency of these modes is very close to their counterparts for $d=5$. However, their growth rate curves do not exhibit the oscillating behaviour observed for finite $d$. These oscillations are associated with the resonance of the trapped boundary modes. This mechanism of resonance has been described in several places (see e.g. Satomura 1981; Yavneh et al. 2001; Gula, Plougonven \& Zeitlin 2009). When the outer boundary is absent, no resonance is possible and the growth is due to another mechanism associated with internal wave emission. This instability mechanism has been described in Le Dizès \& Billant (2009) and Billant \& Le Dizès (2009). Billant \& Le Dizès (2009) have also provided a theoretical dispersion relation for a Rankine vortex which is very similar to (A 7). The differences are only due to the different condition at $r=1$. By contrast with the resonance instability mechanism, the radiative instability concerns a very large band of wavenumbers.

\section{Dependence on the stratification}

In this section, we consider the effect of the stratification. The above results have been obtained for a large value of $N$. In figure 2, we have plotted the characteristics of the most unstable helical mode $(m=1)$ for an unbounded potential Taylor-Couette flow $(\mu=0, d=\infty)$ for $N$ between 0.1 and 5 . We clearly see in figure 2(a) that the growth rate decreases as $N$ decreases below 1 . The progressive stabilization of the radiative instability mode can be associated with the appearance of a critical point in its radial structure as already described in Riedinger et al. (2010a). For the helical mode $(m=1)$, these critical points $r_{c}^{ \pm}$satisfy $\Omega\left(r_{c}^{ \pm}\right)-\omega= \pm N$. The domains in the $(N, \omega)$ plane where such critical points exist in the structure of the mode are delimited 

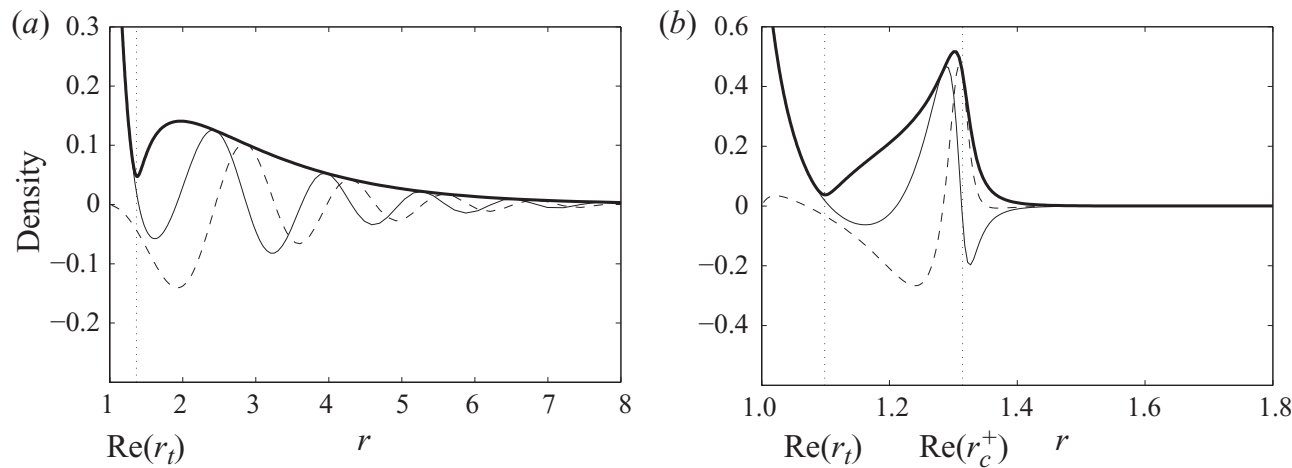

FIGURE 3. Density amplitude (normalized at $r=1)$ of the most unstable helical mode $(m=1)$ for an unbounded potential Taylor-Couette flow $(\mu=0, d=\infty)$. Thick solid line: absolute value; solid line: real part; dashed line: imaginary part. The positions of the real part of the double turning point $r_{t}$ and of the critical point $r_{c}^{+}$are also indicated. (a) $N=1, k=8.7$, $\omega=0.532+0.0461 \mathrm{i} ;$ (b) $N=0.25, k=16, \omega=0.828+0.018 \mathrm{i}$.

by the two dotted lines in figure $2(c)$. This figure indicates that the outer critical point $r_{c}^{+}$is present as soon as $N<0.6$, whereas the inner critical point $r_{c}^{-}$is never present in the most unstable mode structure. The effect of the critical point is visible on the eigenmode structure (see figure 3). Whereas the radiative structure is present up to infinity for large $N$ (figure $3 a$ ), it disappears after $r_{c}^{+}$for small $N$ (figure $3 b$ ).

It is worth mentioning that the instability does not disappear for small $N$. The characteristics of the instability in this limit are provided in Appendix B. We show that for all $m>0$, the most unstable mode becomes localized in an $O(N)$ neighbourhood of the inner cylinder. Its most dangerous wavenumber diverges as $k \sim 4.7 \mathrm{~m} \mathrm{~N}^{-1}$ and its frequency converges to $m$ as $\omega-m \sim(-0.66+0.073 \mathrm{i}) N$. The density amplitude of the most unstable mode in the small $N$ limit is given in figure $9(b)$ of Appendix B. Comparison of this figure with figure $3(b)$ shows that the mode structure for $N=0.25$ is already well described by the small $N$ analysis.

\section{Dependence on the azimuthal wavenumber}

In figure 4, we have plotted the numerical results obtained for $m=1,2,5$ and 10 for an unbounded potential Taylor-Couette flow $(d=\infty, \mu=0)$ for $N=5$. These curves tend to show that both the growth rate and the frequency increase with $m$. This tendency is also in agreement with (A 12) which corresponds to the behaviour for large $m$ of the WKBJ results. However, in this asymptotic theory, $m$ is assumed fixed and finite, so it a priori does not apply.

In the large $m$ limit, a new asymptotic theory has to be constructed. The numerical results tend to show that the unstable modes become more and more localized near the inner boundary as $m$ increases. Based on this observation, it is natural to introduce the following scaling:

$$
\bar{r}=m(r-1), \quad \omega=m+\omega_{0}, \quad k=m N k_{1} .
$$

Moreover, if we assume that $N \gg 1$, which permits replacing $N^{2}-\Phi^{2}$ in (2.4) by $N^{2}$ (this is the hydrostatic hypothesis), (2.4) becomes

$$
\frac{\partial^{2} \psi}{\partial \bar{r}^{2}}-2 \frac{a^{2}\left(\bar{r}-\bar{r}_{o}\right)}{a^{2}\left(\bar{r}-\bar{r}_{o}\right)^{2}-1} \frac{\partial \psi}{\partial \bar{r}}+\left(a^{2}\left(\bar{r}-\bar{r}_{o}\right)^{2}-1-\frac{b}{a^{2}\left(\bar{r}-\bar{r}_{o}\right)^{2}-1}-c\right) \psi=0,
$$



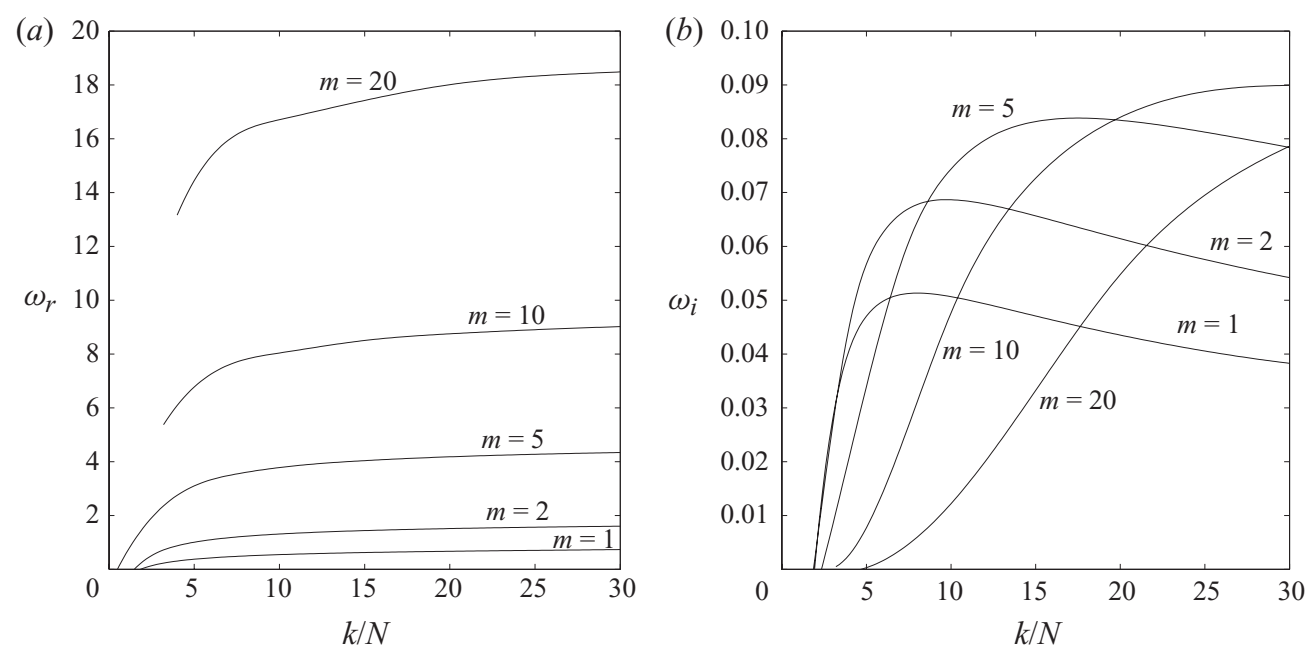

FIGURE 4. Frequency $\omega_{r}(a)$ and growth rate $\omega_{i}(b)$ versus the rescaled axial wavenumber $k / N$ of the most unstable mode of azimuthal wavenumber $m$ in a potential Taylor-Couette flow in an infinite gap $(\mu=0, d=\infty)$ for $N=5$.

where

$$
a=k_{1}\left|\Omega_{1}^{\prime}\right|, \quad b=-2 \Omega_{1}^{\prime} \zeta_{1} k_{1}^{2}, \quad c=2 \Omega_{1} \zeta_{1} k_{1}^{2}, \quad \bar{r}_{0}=\omega_{0} / \Omega_{1}^{\prime} .
$$

This equation is a priori valid for any vortex. The parameters $m$ and $N$ have disappeared from the problem and the mean flow profile now only enters via the values $\Omega_{1}$ and $\Omega_{1}^{\prime}$ of the angular velocity and its radial derivative at $r=1$. This means that the eigenvalue problem now only depends on the local behaviour of the angular velocity near $r=1$. Here, our normalization guarantees that $\Omega_{1}=1$ and that $\Omega_{1}^{\prime}=2 \mu-2$ for Taylor-Couette flows and $\Omega_{1}^{\prime}=\lambda / 2-2$ for Keplerian flows. For large $m$ and large $N$, the stability properties of each flow can then be deduced from the other by changing $\mu \leftrightarrow \lambda / 4$. In particular, the pure Keplerian flow $(\lambda=1)$ has therefore the same stability property as the Taylor-Couette flow with $\mu=1 / 4$ in these limits. It is important to emphasize that the equivalence of both flows is not due to a small-gap approximation. Here the flow is unbounded and only the most dangerous perturbation is localized in a very small region close to the inner boundary.

For the potential flow, we have $a=2 k_{1}, b=c=0, \bar{r}_{0}=\omega_{0} / 2$. The results obtained by integrating (5.2) with these coefficients are compared to the numerical results obtained for $m=1,2,5,10$ and 20 in figure 5. We clearly see that as $m$ increases the numerical results tend to converge to the large $m$ asymptotic theory curve. Note also that the WKBJ theory (defined in (A 12)) predicts correctly the frequency but it fails to provide a good estimate for the growth rate for large $m$.

\section{Taylor-Couette flows in the large-gap limit}

In this section, we consider Taylor-Couette flows for an infinite gap $(d=\infty)$. We first want to analyse how the instability is modified when background rotation is added. We consider Taylor-Couette flow profiles $(2.1 a)$ for $0<\mu$. The growth rate contours obtained by the large $m$ analysis are shown in figure 6 . We clearly see the stabilizing effect of the background rotation. It is difficult to evaluate the critical parameter $\mu$ above which the flow becomes stable because the maximum growth rate 

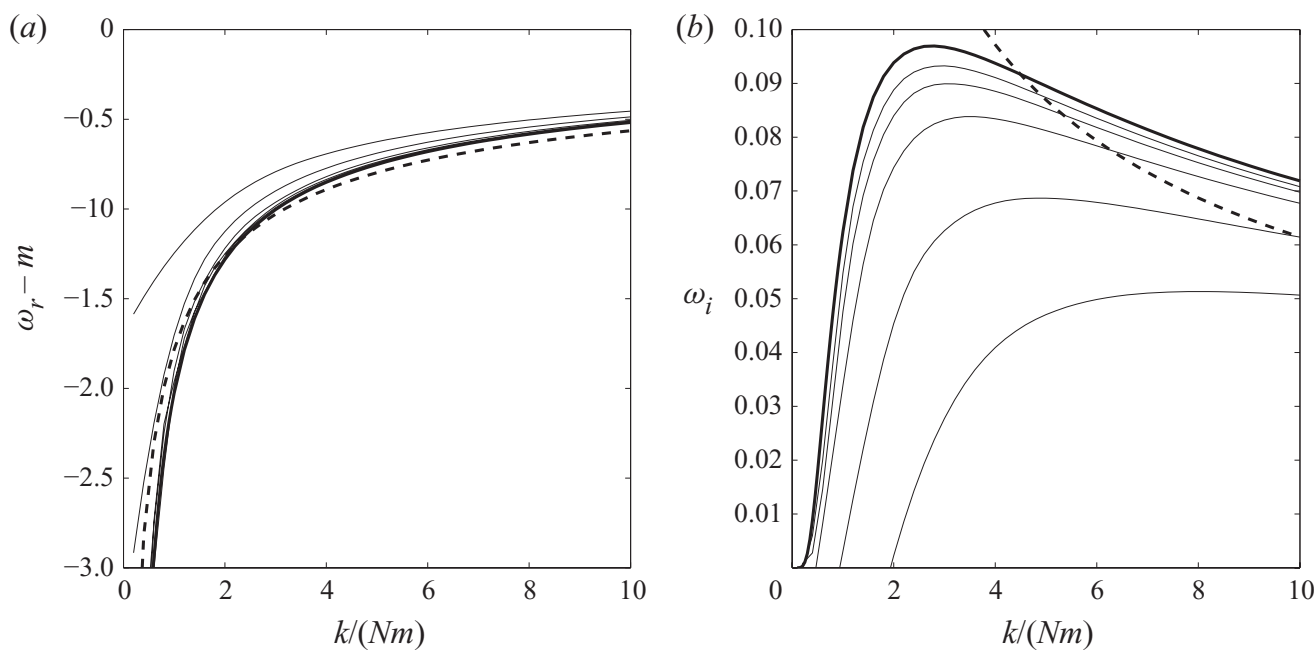

FIGURE 5. Same as figure 4 but relative frequency $\omega_{r}-m(a)$ and growth rate $\omega_{i}(b)$ versus $k /(\mathrm{Nm})$. Thick solid line: large $m$ asymptotic results. Dashed line: WKBJ results for large $m$ (defined in (A 12)). Increasing $m$ are from top to bottom in $(a)$ and from bottom to top in $(b)$.

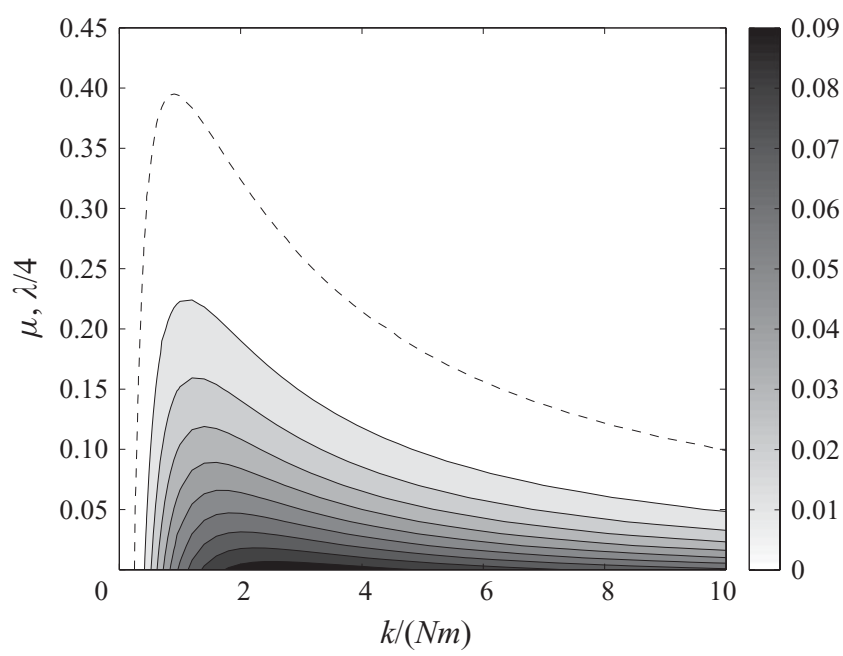

Figure 6. Growth rate contours in the $(k /(\mathrm{Nm}), \mu)$ plane for Taylor-Couette flows obtained by the large $m$ analysis for large $N$. The dashed line corresponds to the $10^{-3}$ contour. This plot also provides the growth rate contours for Keplerian flows where $\mu$ is changed to $\lambda / 4$.

tends to asymptote the zero growth rate curve as $\mu$ increases. Nevertheless, as seen in figure 6 , for $\mu>0.4$ the maximum growth rate becomes smaller than $10^{-3}$, so the radiative instability is not expected to play any role above this value of $\mu$.

The stabilizing effect of the background rotation is present for all values of $m$. In figure $7(a)$, we have plotted the growth rate of the most unstable mode as a function of $\mu$ for several values of $m$ for a strongly stratified case $(N=20)$. We can see that the numerical results converge to the results of the large $m$ analysis as $m$ increases.

The dependence of the stability properties on the strength of the stratification is summarized in figure $7(b)$. In this figure are plotted the contours of the maximum growth rate as both $N$ and $\mu$ are varied for a fixed value of $m$ (here $m=10$ ). 

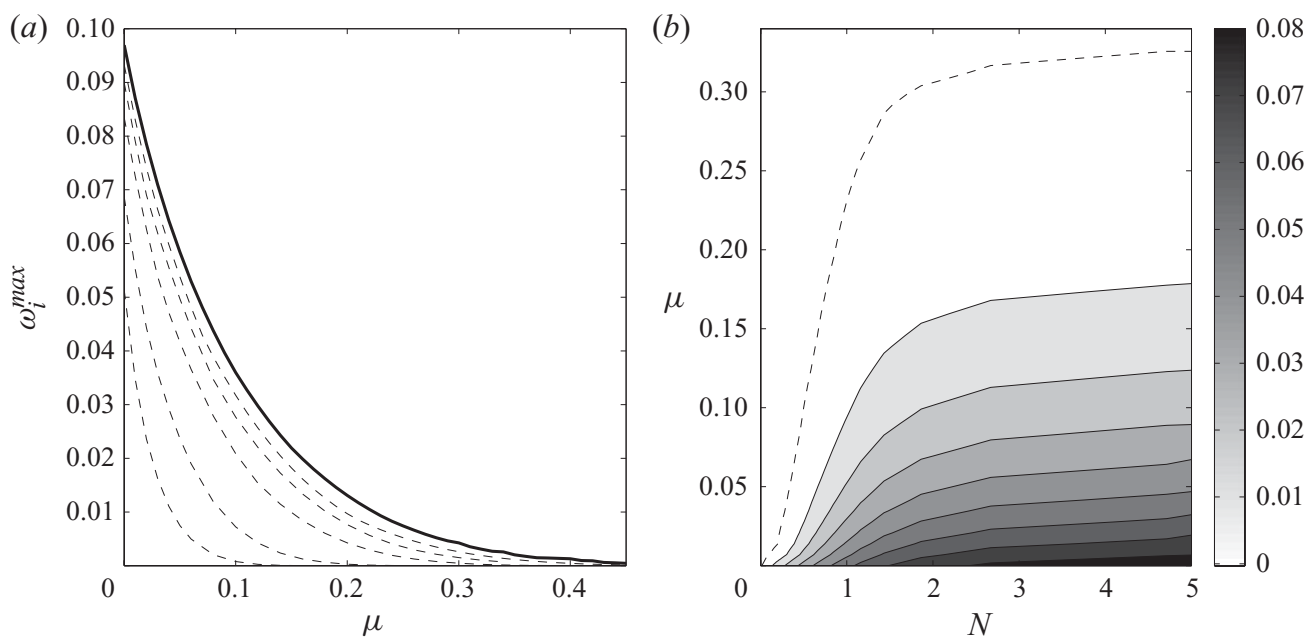

FIGURE 7. Stability properties of Taylor-Couette flows. (a) Maximum growth rate (over $k$ ) versus $\mu$ for $m=1,2,5,10,20$ (dashed lines) for $N=20$. Increasing $m$ are from bottom to top. The prediction obtained by the large $m$ analysis is indicated as a solid line. $(b)$ Maximum growth rate contours in the $(N, \mu)$ plane for $m=10$. The dashed line corresponds to the $10^{-3}$ contour.

Similar contour plots are obtained for other values of $m$. As expected, decreasing the stratification is stabilizing for all $\mu$. The stabilizing mechanism is the same as the one already discussed in $\S 4$. However, when $\mu \neq 0$, the properties of the unstable modes are different for small $N$. The growth rate tends to zero much more rapidly than for $\mu=0$. In the limit of large $m$, we suspect that the instability remains present but with an exponentially small growth rate for small $N$. In practice, we do not expect the instability to play any role when the growth rate becomes smaller than $10^{-3}$. The dashed line shown in figure $7(b)$ can therefore be considered as a good estimate of the marginal stability curve.

\section{Keplerian flows in the large-gap limit}

As mentioned above, for large $N$ and large gaps, the most unstable modes are obtained for large azimuthal wavenumbers and the stability properties of Keplerian flows can be deduced from those of Taylor-Couette flows by changing $\lambda$ to $4 \mu$. Figure 6 thus also provides the maximum growth rate contours of Keplerian flows for large $N$. Keplerian flows are thus expected to be stable for $\lambda \geqslant 1.6$.

It is also interesting to look at the properties of the Keplerian flows for moderate $m$. In figure $8(a)$ are displayed the marginal stability curves of the first azimuthal modes. It shows that, as background rotation for Taylor-Couette flows, the Kepler component of the flow is stabilizing. The first thirteen azimuthal modes become progressively stable as the parameter $\lambda$ is increased. For the pure Keplerian flow $(\lambda=1, d=\infty)$, only the modes with an azimuthal wavenumber larger than or equal to 14 remain unstable. The growth rate contours of the modes in the $(k / N, m)$ plane are shown in figure $8(b)$ for that case. One can notice that the instability growth rates are now much smaller than for the potential Taylor-Couette flow $(\lambda=0)$. Numerical results have also been obtained for different stratifications (not shown). We have obtained that when the stratification is weakened, higher azimuthal wavenumbers also stabilize. When $N$ is smaller than 1 , the growth rate of all the modes is smaller 
(a)

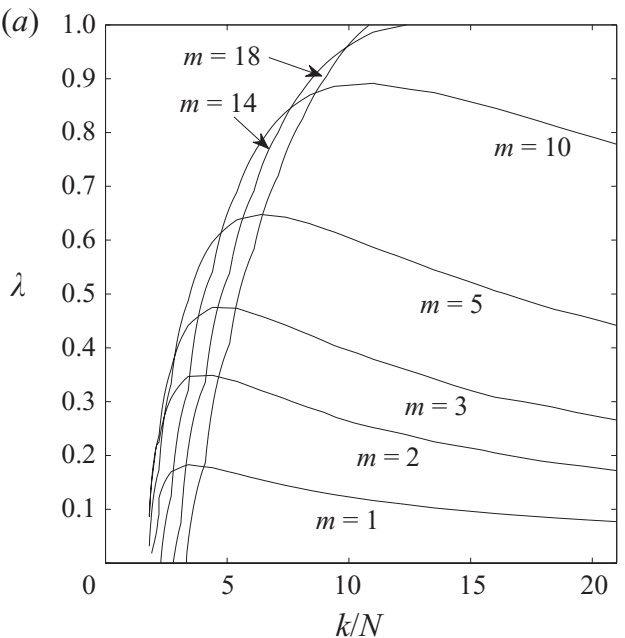

(b)

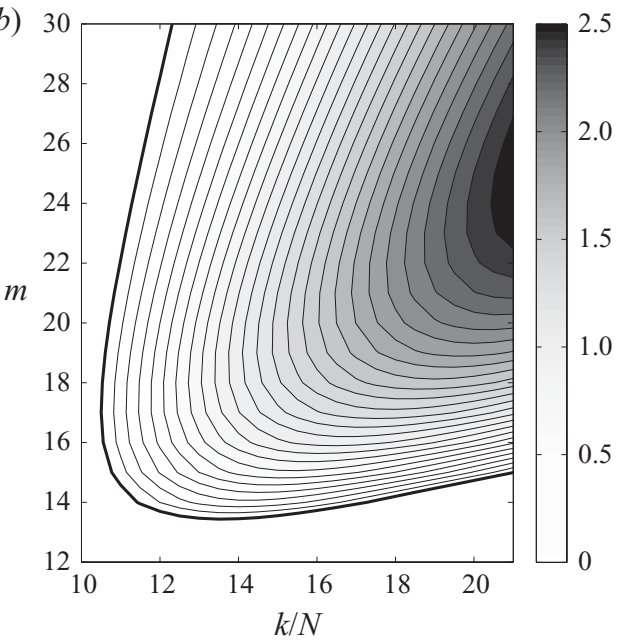

FIGURE 8. Numerical results for Keplerian flows for $N=5$ and $d=\infty$. (a) Marginal stability curves (zero growth rate curves) for different azimuthal wavenumbers in the $(k / N, \lambda)$ plane. (b) Growth rate contours in the $(k / N, m)$ plane for the pure Keplerian flow $(\lambda=1)$.

than $10^{-4}$. In this regime, the pure Keplerian flow $(\lambda=1, d=\infty)$ can be considered as stable.

\section{Conclusion}

We have analysed two families of centrifugally stable rotating flows in the presence of stable stratification. We have first seen that the SRI discovered in a finite gap stratified Taylor-Couette flow (Molemaker et al. 2001; Shalybkov \& Rüdiger 2005; Le Bars \& Le Gal 2007) is transformed into a radiative instability (Le Dizès \& Billant 2009) as the gap size goes to infinity. However, the characteristics of both instabilities are different. Whereas SRI is characterized by small instability bands around specific resonant wavenumbers, the radiative instability concerns (in the inviscid limit) an infinitely large interval of wavenumbers. The transition from one instability to the other occurs by a progressive displacement of the resonant wavenumbers towards the origin and a progressive overlapping of the instability bands. The maximum instability growth rate is also found to decrease, the radiative instability being less severe than the instability in a finite gap.

For an unbounded configuration, we have analysed the sensitivity of the instability to variations of the rotation profile. Whatever the stratification, we have seen that the most unstable profile is the potential flow. Both the addition of a background rotation and of a Keplerian flow component have been found to be stabilizing. For strong stratification (large $N$ ), the maximum growth rate for the Taylor-Couette flows has been shown to become negligible (smaller than $10^{-3}$ ) as soon as $\mu>0.4$. This means that both weak cyclonic and anticyclonic vortices of Rossby number smaller than 1 (in absolute value) are stable with respect to the radiative instability. For large $N$, we have obtained that Keplerian flows are unstable when $\lambda<1.6$. The pure Keplerian flow $(\lambda=1)$ is thus unstable but the maximum growth rate of the instability which is obtained for large $N$ remains small of order $3 \times 10^{-3}$ the maximum angular velocity of the flow. 
The dependence of the radiative instability on the azimuthal wavenumber $m$ has also been analysed. We have shown that the most unstable modes are obtained for large $m$ and that their characteristics are well reproduced by an asymptotic analysis in the limit of large axial and azimuthal wavenumbers. This analysis has also permitted us to show that for large $N$ and large gaps, the most unstable modes are localized near the inner boundary and that the stability properties of Keplerian flows can be deduced from those of Taylor-Couette flows with $\mu=\lambda / 4$. For the pure Keplerian flow, we have also shown that only modes of $m \geqslant 14$ can become unstable.

The effect of the stratification on the instability has also been quantified. We have seen that decreasing the stratification is always stabilizing. In particular, we have observed that the pure Keplerian flow becomes stable for $N$ below 1. In contrast, the potential Taylor-Couette flow remains unstable for small $N$ with a growth rate proportional to $N$. An asymptotic analysis in the limit $N \rightarrow 0$ has provided the structure of the instability mode in this limit.

\section{Discussion}

The results of the present paper can be applied to geophysical vortices and to accretion disks.

The unstable radiative modes obtained here are also present in vortices for which there is no inner boundary. They correspond to the 'ring modes' described in a general framework in Le Dizès \& Billant (2009). As shown in that paper and in Billant \& Le Dizès (2009), they exist for both a Gaussian vortex and a Rankine vortex. Moreover, as shown in Riedinger et al. (2010a), they survive the addition of a weak viscosity. The results for the unbounded Taylor-Couette flow provide information on the effect of background rotation on the radiative instability. We have seen that the instability is present for large $N$ for $\mu<0.4$. This means that it is expected to affect cyclonic vortices of Rossby number $R o>1.5$. The weak anticyclonic vortices $(|R o|<1)$, that is those which are stable with respect to the centrifugal instability, are in contrast not expected to possess the unstable radiative ring modes. A typical atmospheric mesocyclone of diameter $10 \mathrm{~km}$, maximum speed $20 \mathrm{~m} \mathrm{~s}^{-1}$, at mid-latitude $\left(N^{*} \approx 10^{-2} \mathrm{rad} \mathrm{s}^{-1}, f \approx 10^{-4} \mathrm{rad} \mathrm{s}^{-1}\right)$ for which $R o$ and $N$ are 10 and 5, respectively, would therefore be unstable with respect to the radiative instability. In contrast, meddies or other coherent vortices in the oceans for which the Rossby number is in general smaller than 1 would not be affected by radiative ring modes. However, they could be affected by the radiative instability mode which has been filtered out in our analysis, that is, the displacement mode. As such a mode has a longer characteristic wavelength, it is probably necessary to take into account the finite length of the vortex to determine whether it could exist in real vortices.

The source of the momentum transport in accretion disks is still an open question. Numerous possibilities have been put forward ranging from magneto-rotational instability to subcritical shear instability (see e.g. Balbus \& Hawley 1998). The stratorotational instability is among these possibilities as a disk is stratified along its rotation axis. In thin accretion disks, Dubrulle et al. (2005a) have estimated the normalized Brunt-Väisälä frequency $N$ to be 0.3 and have shown using the small-gap Taylor-Couette approximation (or shearing box model) that the pure Keplerian flow should be unstable. We have reached a different conclusion when the unbounded Keplerian profile is used. We have found that the small azimuthal modes $m \leqslant 13$ are all stable in a pure Keplerian flow whatever the stratification and that the higher azimuthal modes $m \geqslant 14$ which are unstable for strong stratification become stable as 
soon as $N<1$. This discrepancy comes from the different nature of the instability in both approaches. The instability modes obtained by Dubrulle et al. (2005a) (see also Molemaker et al. 2001) need the presence of close boundaries as they result from the resonance of boundary trapped modes, whereas our modes exist in an unbounded domain. Note, however, that our modes tend to be localized near the inner boundary. It is therefore not clear how they will be modified with more realistic boundary conditions.

Financial support from the french national agency for research (ANR) under contracts \# BLAN06-3-137005 (FLOWINg Project) and \# ANR-07BLAN-0182 (IMAGINE Project) is gratefully acknowledged. This work has also benefitted from discussions with Paul Billant.

\section{Appendix A. WKBJ analysis of the potential Taylor-Couette flow}

In this appendix, approximate dispersion relations are obtained for the potential Taylor-Couette flow $(\mu=0$ or $\lambda=0)$ by the large-wavenumber WKBJ analysis which has already been used in Le Dizès \& Lacaze (2005) and Le Dizès \& Billant (2009). The idea is to construct eigenmodes by matched asymptotic methods (see e.g. Bender \& Orszag 1978).

For the potential Taylor-Couette flow, the flow domain can be divided into three parts. An inner region localized near the turning point $r_{t}=\sqrt{m / \omega}$ where $\Phi=0$ and two outer regions $O^{ \pm}$located on either side of $r_{t}$.

In the outer regions, the solution can be described in terms of WKBJ approximations (Bender \& Orszag 1978). Applying the boundary condition at $r=1$ implies that, in the region $O^{-}$between 1 and $r_{t}$, the solution can be written as

$$
\Psi^{-}=A Q(r) \sin \left(\int_{1}^{r} \beta\right)
$$

with

$$
\beta=k \frac{\Phi}{\sqrt{N^{2}-\Phi^{2}}}, \quad Q(r)=r^{1 / 2} G^{1 / 4} .
$$

Similarly, the solution in the region $O^{+}$between $r_{t}$ and $d$ can be written as

$$
\Psi^{+}=B Q(r) \sin \left(\int_{d}^{r} \beta\right),
$$

when a boundary is present at $r=d$ and as

$$
\Psi^{+}=B Q(r) \exp \left(\mathrm{i} \int_{r_{t}}^{r} \beta\right),
$$

when there is no outer boundary.

The dispersion relation is obtained by matching these solutions with the solution in the inner region which is described by the following equation:

$$
\frac{\partial^{2} \Psi}{\partial \tilde{r}^{2}}-\frac{2}{\tilde{r}} \frac{\partial \Psi}{\partial \tilde{r}}+\alpha^{2} \tilde{r}^{2} \Psi=0
$$

with $\tilde{r}=k\left(r-r_{t}\right)$ and $\alpha=\left|m \Omega_{t}^{\prime} / N\right|$. Writing the solution of (A 5) as

$$
\Psi(\tilde{r})=\tilde{r}^{3 / 2}\left[\tilde{A} H_{3 / 4}^{(1)}\left(\alpha \tilde{r}^{2}\right)+\tilde{B} H_{3 / 4}^{(2)}\left(\alpha \tilde{r}^{2}\right)\right],
$$


we easily obtain from the behaviour of the Hankel functions at $\infty$, the condition of matching leading to the dispersion relations

$$
K_{1 t}^{2}=\mathrm{i} \sqrt{2},
$$

when $d=\infty$ and

$$
\left(K_{1 t}^{2}-\mathrm{i} \sqrt{2}\right)\left(K_{t d}^{2}-\mathrm{i} \sqrt{2}\right)=-K_{1 t}^{2} K_{t d}^{2}
$$

when $d$ is finite, where

$$
K_{1 t}=\exp \left(\mathrm{i} \int_{1}^{r_{t}} \beta\right), \quad K_{t d}=\exp \left(\mathrm{i} \int_{r_{t}}^{d} \beta\right) .
$$

In the strongly stratified limit, the dispersion relation can be further simplified because in this limit

$$
K_{1 t} \sim \exp \left(\mathrm{i} \frac{k \omega}{N}\left(\sqrt{\frac{m}{\omega}}-1\right)^{2}\right), \quad K_{t d} \sim \exp \left(\mathrm{i} \frac{k \omega}{d N}\left(d-\sqrt{\frac{m}{\omega}}\right)^{2}\right) .
$$

In particular, (A 7) can be written explicitly as

$$
\omega=\left[\sqrt{\frac{N}{k}}\left(n \pi+\frac{\pi}{4}-i \frac{\log (2)}{4}\right)^{1 / 2}+\sqrt{m}\right]^{2},
$$

where $n$ is an integer.

Note finally that for large $m$, this expression reduces to

$$
\omega-m \sim 2 \sqrt{\frac{N m}{k}}\left(n \pi+\frac{\pi}{4}-\mathrm{i} \frac{\log (2)}{4}\right)^{1 / 2} .
$$

\section{Appendix B. Small $N$ analysis of the unbounded potential Taylor-Couette flow}

As $N$ goes to zero, the most unstable modes of the unbounded potential flow ( $\mu=0$, $d=\infty$ ) tend to become more and more localized near the inner cylinder at $r=1$. We therefore expect that the structure of these modes can be captured by performing a local asymptotic analysis as $N$ goes to zero of the solution near $r=1$. The procedure of such an analysis is classical (see Bender \& Orszag 1978) and is based on the use of a local variable.

Here, for any fixed $m>0$, the appropriate change of variables is

$$
y=\frac{m \Omega_{1}^{\prime}(1-r)}{N}+\omega_{1}, \quad \omega=m+\omega_{1} N, \quad k=\frac{k_{o} m\left|\Omega_{1}^{\prime}\right|}{N},
$$

where $\Omega_{1}^{\prime}=-2$ for the potential flow. It allows us to transform (2.4) at leading order in $N$ as $N \rightarrow 0$ to an equation which only depends on the parameter $k_{o}$ :

$$
\frac{\partial^{2} \psi}{\partial y^{2}}-\frac{2}{y\left(1-y^{2}\right)} \frac{\partial \psi}{\partial y}+\frac{k_{o} y^{2}}{1-y^{2}} \psi=0
$$

This equation has to be solved with the condition that $\psi$ vanishes as $y$ goes to infinity, such that the solution is indeed localized near the inner cylinder. The boundary condition at $r=1$, which becomes $\psi\left(\omega_{1}\right)=0$, then provides $\omega_{1}$.

This eigenvalue problem is solved by a shooting method. The dependence of the imaginary part of the most unstable eigenvalue $\omega_{1}$ on $k_{o}$ is shown in figure $9(a)$. 

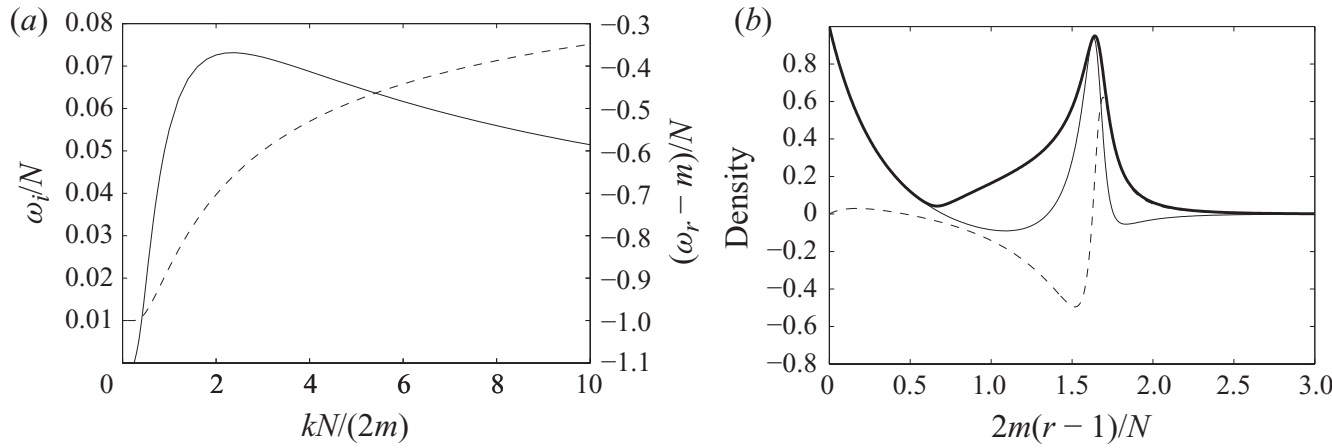

FIGURE 9. Stability properties of the unbounded potential flow $(\mu=0, d=\infty)$ for small $N$. (a) Rescaled growth rate $\omega_{i} / N$ (left axis and solid line) and relative frequency $\left(\omega_{r}-m\right) / N$ (right axis and dashed line) versus $k_{o}=k N /(2 m)$. (b) Density fluctuation (normalized at $r=1$ ) of the most unstable mode (obtained for $k_{o} \approx 2.35$ ). Thick solid line: absolute value, solid line: real part, dashed line: imaginary part.

The most unstable mode is obtained for $k_{o}^{\max } \approx 2.35$ and has the following frequency: $\omega_{1}^{\max } \approx-0.66+0.073 \mathrm{i}$. The radial structure of the density amplitude associated with the most unstable eigenmode is plotted in figure $9(b)$.

\section{REFERENCES}

Balbus, S. A. \& Hawley, J. F. 1998 Instability, turbulence, and enhanced transport in accretion disks. Rev. Mod. Phys. 70 (1), 1-53.

Bender, C. M. \& Orszag, S. A. 1978 Advanced Mathematical Methods for Scientists and Engineers. McGraw-Hill.

Billant, P \& Gallaire, F. 2005 Generalized Rayleigh criterion for non-axisymmetric centrifugal instabilities. J. Fluid Mech. 542, 365-379.

Billant, P. \& Le Dizès, S. 2009 Waves on a columnar vortex in a strongly stratified fluid. Phys. Fluids 21, 106602.

Dubrulle, B., Dauchot, O., Daviaud, F., Longaretti, P.-Y., Richard, D. \& Zahn, J.-P. $2005 a$ Stability and turbulent transport in Taylor-Couette flow from analysis of experimental data. Phys. Fluids 17, 095103.

Dubrulle, B., Marie, L., Normand, C., Richard, D., Hersant, F. \& Zahn, J.-P. $2005 b$ A hydrodynamic shear instability in stratified disks. Astron. Astrophys. 429, 1-13.

Goldreich, P. \& Lynden-Bell, D. 1965 Spiral arms as sheared gravitational instabilities. Mon. Not. R. Astron. Soc. 130, 125-158.

Gula, J., Plougonven, R. \& Zeitlin, V. 2009 Ageostrophic instabilities of fronts in a channel in a stratified rotating fluid. J. Fluid Mech. 627, 485-507.

Hopfinger, E. J. \& van Heisst, G. J. F. 1993 Vortices in rotating fluids. Annu. Rev. Fluid Mech. 25, 241-289.

Le Bars, M. \& Le Gal, P. 2007 Experimental analysis of the stratorotational instability in a cylindrical Couette flow. Phys. Rev. Lett. 99, 064502.

Le Dizès, S. \& Billant, P. 2009 Radiative instability in stratified vortices. Phys. Fluids 21, 096602.

Le Dizès, S. \& LaCaze, L. 2005 An asymptotic description of vortex Kelvin modes. J. Fluid Mech. 542, 69-96.

Molemaker, M. J., McWilliams, J. C. \& Yavneh, I. 2001 Instability and equilibration of centrifugally stable stratified Taylor-Couette flow. Phys. Rev. Lett. 86, 5270-5273.

Narayan, R., Goldreich, P. \& Goodman, J. 1987 Physics of modes in a differentially rotating system - analysis of the shearing sheet. Mon. Not. R. Astron. Soc. 228, 1-41.

Riedinger, X., Le Dizès, S. \& Meunier, P. 2010a Viscous stability properties of a Lamb-Oseen vortex in a stratified fluid. J. Fluid Mech. 645, 255-278. 
Riedinger, X., Meunier, P. \& Le Dizès, S. $2010 b$ Instability of a vertical columnar vortex in a stratified fluid. Exp. Fluids (in press, doi:10.1007/S00348-010-0833-0 online).

Satomura, T. 1981 An investigation of shear instability in a shallow water. J. Meteorol. Soc. Japan 59, $148-167$.

Schecter, D. A. \& Montgomery, M. T. 2004 Damping and pumping of a vortex Rossby wave in a monotonic cyclone: critical layer stirring versus inertia-buoyancy wave emission. Phys. Fluids 16, 1334-1348.

Shalybkov, D. \& Rüdiger, G. 2005 Non-axisymmetric instability of density-stratified TaylorCouette flow. J. Phys.: Conf. Ser. 14, 128-137.

Withuack, E. M. \& Chen, C. F. 1974 An experimental study of Couette instability of stratified fluids. J. Fluid Mech. 66, 725-737.

Yavneh, I., McWilliams, J. C. \& Molemaker, M. J. 2001 Non-axisymmetric instability of centrifugally stable stratified Taylor-Couette flow. J. Fluid Mech. 448, 1-21. 